lymphadenopathy in $63 \%$, and cardiomyopathy in $30 \%$. Failure to gain weight predated the onset of encephalopathy in infected infants. HIV infected children without encephalopathy had a lower incidence of hepatosplenomegaly (29\%) and cardiomyopathy (2\%), diagnosed in the first 3 months of life. Risk of encephalopathy was related to a high viral load in infancy. (Cooper ER, Hanson C, Diaz C et al. Encephalopathy and progression of human immunodeficiency virus disease in a cohort of children with perinatally acquired human immunodeficiency virus infection. I Pediatr May 1998;132:808-812). (Reprints: Ellen R Cooper MD, Finland Laboratory, Boston Medical Center, 774 Albany St, Boston, MA 02118).

COMMENT. Encephalopathy is a frequent complication of perinatally acquired HIV infection, occurring in one in 5 infants. A high viral load during the neonatal period, failure to thrive, and early signs of organomegaly and lymphadenopathy are risk factors for HIV encephalopathy.

\title{
NEUROLOGIC COMPLICATIONS OF E. COLI H-UREMIC SYNDROME
}

The association between bacterial genotype of E coli 0157:H7 and CNS manifestations of childhood gastroenteritis-associated hemolytic uremic syndrome (D+HUS) was studied in 51 patients with HUS treated at the British Columbia's Children's Hospital, Vancouver, Canada. Of 51 children with HUS, 11 suffered neurologic complications that included encephalopathy in 6 and seizures in 7, with 2 deaths. No association with bacterial genotype was demonstrated. (Cimolai N, Carter JE. Bacterial genotype and neurological complications of Escherichia coli 0157:H7-associated haemolytic uraemic syndrome. Acta Paediatr May 1998;87:593-594). (Respond: Dr N Cimolae, Room 2G6, Department of Pathology and Laboratory Medicine, British Columbia's Children's Hospital, 4480 Oak Street, Vancouver, British Columbia, Canada).

COMMENT: Seizures, encephalopathy, visual disturbances, and transient hemiparesis are the neurologic complications of $\mathrm{E}$ coli hemolytic uremic syndrome. Seizures, the most common symptom, occur from 4 to 12 days after onset of diarrhea. A number of factors may cause the CNS symptoms, but a specific bacterial genotype of E coli is not implicated. Prevention or timely diagnosis and treatment may diminish the incidence of neurologic complications of $\mathrm{E}$ coli 0157:H7 infection.

Risk of hemolytic uremic syndrome after sporadic E coli O157:H7 infection: Results of a Canadian collaborative study. (Rowe PC et al. I Pediatr May $1998 ; 132: 777-782)$. Of 205 children with HUS, $77 \%$ had E coli $0157:$ H7 infection. A further 582 had E coli gastroenteritis. The risk of HUS in Alberta was 8.1\%, compared to $31 \%$ in other Canadian referral centers.

\section{SEIZURE DISORDERS}

\section{COGNITIVE AND BEHAVIORAL OUTCOMES OF FEBRILE SEIZURES}

Of approximately 14,000 children enrolled in a British Child Health and Education Study, 398 identified with febrile convulsions (FC) were assessed at age 10 years at Addenbrooke's Hospital, Cambridge, and the University of Bristol, UK. Measures of academic progress, intelligence, and behavior in the FC patients were not significantly different from controls without FC. Patients with simple FC (287) and complex FC (94) showed similar results. The outcomes in those with recurrent FC and those with a single seizure were similar. Children with FCs in the first year of life required special schooling more often than those with late-onset FCs $(7.5 \%$ 